\title{
Inhibition of the p38 MAPK pathway sensitizes human gastric cells to doxorubicin treatment in vitro and in vivo
}

\author{
WEI TAN ${ }^{1}$, HONG-GANG YU ${ }^{2}$ and HE-SHENG LUO ${ }^{2}$ \\ ${ }^{1}$ Department of Gastroenterology, Wuhan University, Renmin Hospital; ${ }^{2}$ Institute for Gastroenterology and Hepatology, \\ Wuhan University Medical School, Wuhan, Hubei 430060, P.R. China
}

Received October 23, 2013; Accepted May 15, 2014

DOI: $10.3892 / \mathrm{mmr} .2014 .2598$

\begin{abstract}
Gastric cancer is the second most common cause of cancer-related deaths worldwide. Doxorubicin-based chemotherapeutic regimes have been the mainstay of systemic treatment for disseminated gastric cancer for numerous years. However, the efficacy of doxorubicin is severely limited due to chemoresistance. Chemoresistance is a tightly regulated process, under the control of numerous signal transduction pathways. Amongst these, the mitogen-activated protein kinase (MAPK) pathway has received much attention. This study assessed whether the p38 MAPK pathway is involved in doxorubicin resistance in gastric cancer cells. Doxorubicin alone or combined with the p38 MAPK pathway inhibitor SB203580 was used to treat gastric cancer cells (SGC7901 and BGC823 lines). The effect of doxorubicin on the growth and apoptosis of gastric cancer cells in the presence or absence of SB203580 was investigated by western blot analysis, 3-(4,5-dimethylthiazol-2-yl)-2,5-diphenyltetrazolium bromide (MTT) assay, Hoechst staining, Annexin V-FITC/propidium iodide staining followed by flow cytometry analysis, and the terminal deoxynucleotidyl transferase-mediated dUTP nick end labeling (TUNEL) assay. Next, the effects of doxorubicin and SB203580, on the sensitivity of BGC-823 cells were assessed in a tumor xenograft model. The results showed that the p38 MAPK inhibitor significantly increases gastric cancer cell sensitivity to doxorubicin. Doxorubicin in combination with SB203580 significantly reduced cell viability $(\mathrm{P}<0.01)$ and increased cell death $(\mathrm{P}<0.01)$, which may be associated with the inactivation of the p38 MAPK signaling pathway, followed by the induced expression of the pro-apoptotic protein Bax and a concomitant decrease in Bcl-2 expression. These findings suggest that p38 MAPK is involved in gastric cancer cell survival, and that
\end{abstract}

Correspondence to: Miss. Wei Tan, Department of Gastroenterology, Wuhan University, Renmin Hospital, 238 Jiefang Road, Wuchang, Wuhan, Hubei 430060, P.R. China

E-mail: polo5352877@163.com

Key words: gastric cancer, doxorubicin, p38 MAPK, chemoresistance the inhibition of p38 MAPK signaling can reduce the tolerance of gastric cancer cells to doxorubicin treatment.

\section{Introduction}

Gastric cancer is a major health issue and has the second highest cancer-related mortality rate in the world (1). Countries in East Asia such as China, Japan and Korea, have the highest gastric cancer incidence, with an estimated one million new cases reported in 2011 (2). Complete tumor resection is the standard care for gastric cancer, but recurrence is frequent (3). Thus, chemotherapy is important to increase the survival of patients with gastric cancer (4). Doxorubicin, which induces DNA double-strand breaks by interfering with the activity of topoisomerase II- $\alpha$ (5), has been broadly applied for chemotherapy of solid cancers, including gastric cancer. However, the efficacy of doxorubicin is limited by resistance mechanisms that hinder its clinical success (6). A factor that contributes to the poor outcome of chemotherapy is chemoresistance (7). It was demonstrated that chemoresistance is a tightly regulated process, under the control of numerous signal transduction pathways (8). Amongst these, the p38 mitogen-activated protein kinase (MAPK) pathway has received much attention (9).

p38, a class of serine/threonine MAPK, are activated through phosphorylation at the Thr180-Gly-Tyr182 motif by MKK3, MKK4 and MKK6 (10). The p38 MAPK pathway activates a wide range of substrates that include transcription factors, protein kinases, and nuclear proteins, leading to diverse responses, including anti-apoptotic and chemoresistance processes (11). p38 MAPK was found to be abberantly activated in gastric cancer cells (12).

To date, there have been no studies regarding the association of p38 MAPK with the sensitivity of gastric cancer cells to doxorubicin. In this study, we treated gastric cancer cells with doxorubicin with or without SB203580, a specific p38 MAPK inhibitor, and assessed the effects of the treatment on cell survival, apoptosis, and tumor growth. We specifically enquired and confirmed that the p38 MAPK inhibitor can enhance doxorubicin chemosensitivity in two gastric cancer cell lines (SGC7901 and BGC823) by inducing apoptosis in vitro and in vivo, which suggested that p38 MAPK may be considered as a valid molecular target in combination with doxorubicin for the therapeutic treatment of patients with gastric cancer. 


\section{Materials and methods}

Cell cultures. The BGC823, SGC7901, MGC803 and HGC27 cell lines were purchased from the Cell Bank of the Shanghai Institute of Biochemistry and Cell Biology at the Chinese Academy of Sciences. The cells were cultured in Gibco ${ }^{\circledR}$ RPMI-1640 medium (Thermo Fisher Scientific, Waltham, MA, USA) supplemented with heat-inactivated $10 \%$ fetal bovine serum (FBS), $10 \mathrm{U} / \mathrm{ml}$ penicillin and $10 \mu \mathrm{g} / \mathrm{ml}$ streptomycin, in a humidified atmosphere $\left(5 \% \mathrm{CO}_{2}\right.$ and $95 \%$ air) at $37^{\circ} \mathrm{C}$.

Antibodies and reagents. Doxorubicin was purchased from Alexis Corp. (Lausen, Switzerland). SB203580 was purchased from Santa Cruz Biotechnology, Inc. (Santa Cruz, CA, USA) The primary antibodies against human p38 and phosphorylated (phospho)-p38 were purchased from Cell Signaling Technology, Inc. (Beverly, MA, USA), and those against B-cell lymphoma 2 (Bcl-2) and Bcl-2-associated X protein (Bax) were purchased from Santa Cruz Biotechnology, Inc. (Santa Cruz, CA, USA).

3-(4,5-dimethylthazol-2-yl)-2,5-diphenyltetrazolium bromide (MTT) assay. Gastric cancer cells were seeded into 96-well plates $\left(6.0 \times 10^{3}\right.$ cells/well) and were allowed to attach overnight. Following adhesion, freshly prepared doxorubicin at the appropriate concentration $(0,0.3$ and $3 \mu \mathrm{M})$, supplemented with or without SB203580 (20 $\mu \mathrm{M}, 2 \mathrm{~h}$ pretreatment) was added to the cells for different time periods $(0,3,6,12$ and $24 \mathrm{~h}$ ). The viability of cells was evaluated using an MTT assay according to the manufacturer's instructions (Roche Applied Science, Indianapolis, IN, USA). Briefly, MTT was added at a concentration of $500 \mathrm{mg} / \mathrm{l}$, and the cells were incubated for $4 \mathrm{~h}$ at $37^{\circ} \mathrm{C}$. The absorbance of each well was measured at a wavelength of $570 \mathrm{~nm}$ using a computer-controlled microtiter plate reader (Greiner Labortechnik, Frickenhausen, Germany). Cell growth inhibitory rates were defined as the relative absorbance of treated vs. that of the untreated cells.

Hoechst staining. BGC823 cells were cultured in 6-well tissue culture plates and treated with $0.3 \mu \mathrm{M}$ doxorubicin supplemented with or without SB203580 for $12 \mathrm{~h}$. Next, Hoechst 33258 (Sigma-Aldrich, St. Louis, MO, USA) was added to the culture medium; changes in nuclear morphology were examined under a fluorescence microscope (Nikon, Tokyo, Japan) with a $365 \mathrm{~nm}$ filter, appropriate for visualizing Hoechst 33258. The number of Hoechst-positive nuclei per optical field (minimum 50 fields) was counted.

Flow cytometric analysis of apoptosis. BGC 823 cells were incubated in the presence of doxorubicin $(0.3 \mu \mathrm{M})$ supplemented with or without SB203580 for an additional $12 \mathrm{~h}$, then harvested and fixed with $2.5 \%$ glutaraldehyde for $30 \mathrm{~min}$, and washed twice with ice-cold phosphatebuffered saline (PBS). Apoptotic cells in the BGC823 group (DMSO), doxorubicin group (3), SB20450 group and doxorubicin with SB203580 group were detected using a flow cytometer (Molecular Probes ${ }^{\circledR}$; Thermo Fisher Scientific) following Annexin V-fluorescein isothiocyanate (FITC) and propidium iodide (PI) staining (BioVision, Mountain View, CA, USA).
Western blot assay. Protein expression levels were analyzed by western blot analysis. Briefly, the cells were washed with PBS (pH 7.4) and lysed with lysis buffer (20 mM Tris- $\mathrm{HCl}$, $\mathrm{pH} 7.4,150 \mathrm{mM} \mathrm{NaCl}, 0.5 \%$ Nonidet P-40, $1 \mathrm{mM}$ EDTA, $50 \mu \mathrm{g} / \mathrm{ml}$ leupeptin, $30 \mu \mathrm{g} / \mathrm{ml}$ aprotinin, and $1 \mathrm{mM}$ phenylmethylsulfonyl fluoride). Then, cell lysates were quantified using a BCA Protein Assay kit (Thermo Fisher Scientific) and loaded at a concentration of $40 \mu \mathrm{g} /$ lane, separated on a $12.5 \%$ sodium dodecyl sulfate polyacrylamide gel by electrophoresis (SDS-PAGE), and transferred onto a nitrocellulose membrane (Millipore, Bedford, MA, USA) using a wet transfer system (Bio-Rad, Hercules, CA, USA). Next, the membrane was blocked with $10 \%$ nonfat dry milk in Tris-buffered saline with Tween-20 (TBST, pH 8.0) and incubated overnight at $4^{\circ} \mathrm{C}$ with the primary polyclonal rabbit anti-human anti-p38, -phospho-p38, -Bcl-2,-Bax and -actin. The appropriate horseradish peroxidase (HRP)-conjugated goat anti-rabbit secondary antibodies (Cell Signaling Technology, Inc.) were then added at a 1:3,000 dilution for $2 \mathrm{~h}$. Positive reactions were detected with an enhanced Phototope TM-HRP detection kit (Cell Signaling Technology, Inc.) and a hyperfilm X-ray film (Carestream Health, Inc., Rochester, NY, USA).

Caspase-3 activity assay. A caspase-3 Colorimetric assay kit (Nanjing KeyGen Biotech Co., Ltd., Nanjing, China) was used to measure the actitivy of caspase-3. According to the manufacturer's instructions, the cells were seeded into a 6-well plate and cultured for $24 \mathrm{~h}$. The cells were then incubated in the presence of doxorubicin $(0.3 \mu \mathrm{m})$ supplemented with or without SB203580 for an additional $12 \mathrm{~h}$. The cells were then harvested, resuspended in $50 \mu \mathrm{l}$ lysis buffer and incubated on ice for $30 \mathrm{~min}$. The cellular debris was then pelleted and the lysates (50 $\mu 1$ of $2 \mathrm{X}$ reaction bugger with $5 \mu \mathrm{l}$ caspase-3 substrate) and incubated for $4 \mathrm{~h}$ at $37^{\circ} \mathrm{C}$ in a $5 \% \mathrm{CO}_{2}$ incubator.The activities were quantified spectrophotometrically at a wavelength of $405 \mathrm{~nm}$ using the computer-controlled microtiter plate reader. The DMSO group was normalized.

In vivo assessment of tumor growth. All animal procedures were approved by the Committee on Animal Experimentation of Wuhan University, and the procedures complied with the NIH Guide for the Care and Use of Laboratory Animals. BGC823 cells were injected into the flanks of BALB/c nude mice $(\mathrm{Nu} / \mathrm{Nu}$, female, 4-6 weeks old), purchased from the Center of Experimental Animals of Wuhan University and maintained under pathogen-free conditions. Nude mice were randomly divided into four groups: i) the BGC823 group (control); ii) the doxorubicin group ( $2 \mathrm{mg} / \mathrm{kg})$; iii) the SB203580 group $(10 \mathrm{mg} / \mathrm{kg})$; and iv) the doxorubicin $(2 \mathrm{mg} / \mathrm{kg})+\mathrm{SB} 203580(10 \mathrm{mg} / \mathrm{kg})$ group. Tumor growth was monitored by measuring tumor volume, which was calculated by the formula: $\mathrm{V}\left(\mathrm{mm}^{3}\right)=\operatorname{width}^{2}\left(\mathrm{~mm}^{2}\right) \mathrm{x}$ length $(\mathrm{mm}) / 2$. At the end of the experiment, the tumors were harvested for further analyses, described below.

Terminal deoxynucleotidyl transferase-mediated dUTP nick end labeling (TUNEL) assay. Each group of nude mice was weighed at the end of the experiment. Next, to detect apoptotic cells in tumor tissue sections, an In Situ Apoptosis Detection kit-POD (Roche Applied Science, Indianapolis, IN, 
A
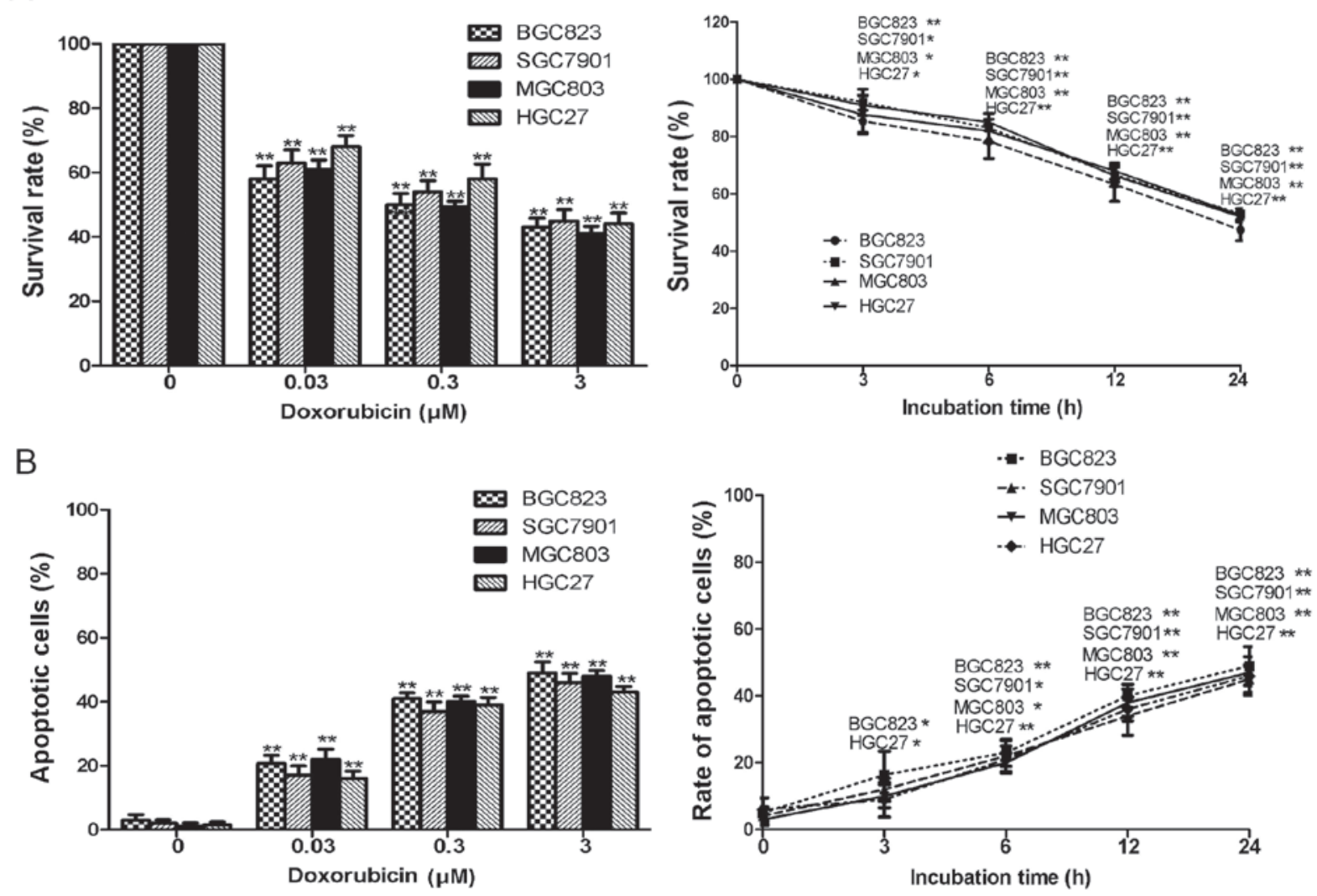

Figure 1. Effect of doxorubicin on growth and apoptosis of gastric cancer cells. (A) Survival rate of the 4 gastric cancer cell lines BGC823, SGC7901 MGC803 and HGC27, treated with different concentrations of doxorubicin for $12 \mathrm{~h}$ (left panel), and treated with $0.3 \mu \mathrm{M}$ doxorubicin for $0,3,6,12$ and $24 \mathrm{~h}$ (right panel), as measured by the MTT assay. (B) Percentage of apoptotic cells in the 4 gastric cancer cell lines, treated with different concentrations of doxorubicin for $12 \mathrm{~h}$ (left), and treated with $0.3 \mu \mathrm{M}$ doxorubicin for $0,3,6,12$ and $24 \mathrm{~h}$ (right), as measured by flow cytometry analysis on Annexin V-fluorescein isothiocyanatepropidium iodide-stained cells. ${ }^{*} \mathrm{P}<0.05$ and ${ }^{* *} \mathrm{P}<0.01$ vs. 0 .

A

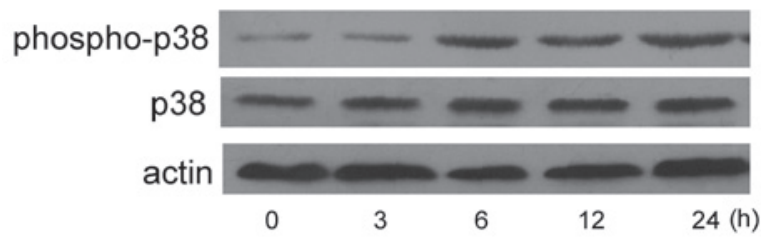

B

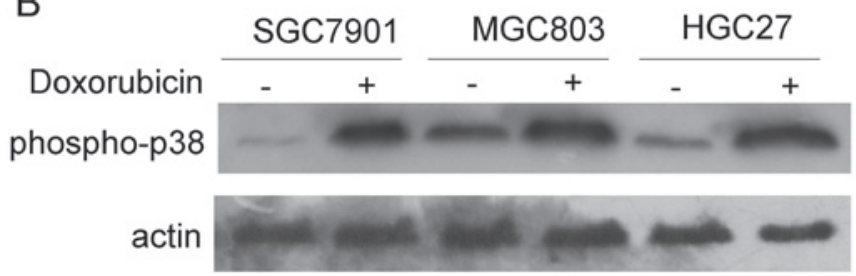

Figure 2. Doxorubicin-induced expression of phosphorylated p38 (phospho-p38) in human gastric cancer cells, as determined by western blot analysis. (A) BGC823 cells were incubated with $0.3 \mu \mathrm{M}$ doxorubicin for the indicated time periods. (B) The indicated human gastric cancer cell lines were incubated with dimethyl sulfoxide (-) or $0.3 \mu \mathrm{M}$ doxorubicin (+) for $12 \mathrm{~h}$ Actin was used as the loading control in all experiments. Three independent repeats were performed.

USA) was used. Tumor sections were dewaxed with dimethylbenzene, rehydrated in a gradient of ethanol, incubated with proteinase $\mathrm{K}$, and rinsed with $\mathrm{ddH}_{2} \mathrm{O}$. A $3 \% \mathrm{H}_{2} \mathrm{O}_{2}$ solution was used to block the endogenous peroxidase activity. Following incubation with the equilibration buffer and the terminal deoxynucleotidyl transferase enzyme, the sections were incubated with the anti-digoxigenin-peroxidase conjugate. The peroxidase activity in each section was detected using diaminobenzidine. Finally, the sections were counterstained with hematoxylin and eosin. Positive cells were identified and counted (three random fields per slide) under a light microscope (Carl Zeiss, Thornwood, NY, USA).

Statistical analysis. Data were expressed as the mean \pm standard error of the mean (SEM). The means of the different groups were compared using a one-way analysis of variance (ANOVA). All statistical analyses were performed with the SPSS 13.0 software (SPSS Inc., Chicago, IL, USA). Statistical significance was accepted at $\mathrm{P}<0.05$.

\section{Results}

Doxorubicin inhibits cell growth and induces apoptosis in gastric cancer cells. The MTT assay was used to assess the effect of doxorubicin on growth of the gastric cancer cell lines MGC803, BGC823, SGC7901 and HGC27. Doxorubicin reduced the growth rates of cells in all 4 lines in a dose- and timedependent manner (Fig. 1A). In addition, Annexin V-FITC/PI staining followed by flow cytometry ${ }^{\text {analysis }}$ was used to detect 

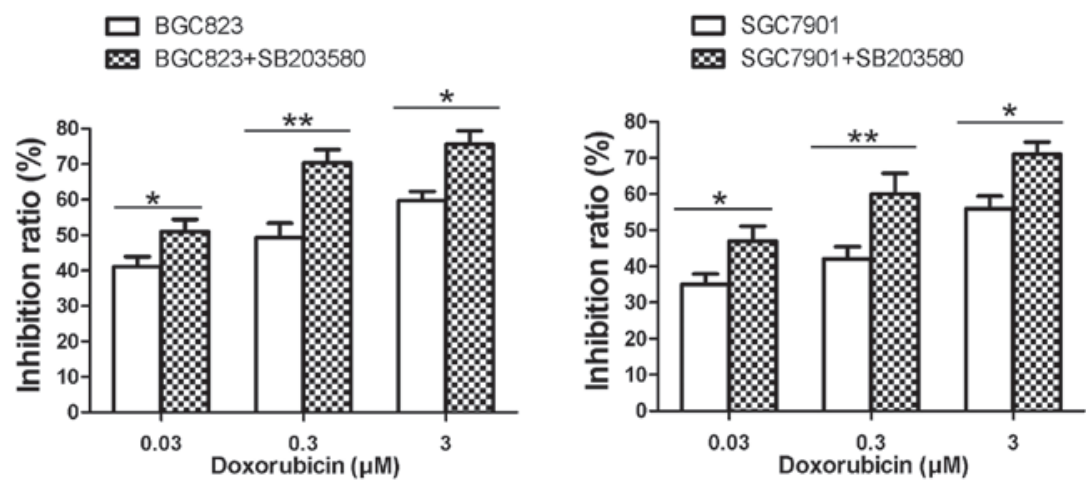

Figure 3. The ratio of cellular growth inhibition in gastric cancer cells, as measured by the MTT assay. BGC823 and SGC7901 cells were treated with different concentrations of doxorubicin, supplemented with or without SB203580 (20 $\mu \mathrm{M}, 2$ h pretreatment $)$ for $12 \mathrm{~h} .{ }^{*} \mathrm{P}<0.05$ and ${ }^{* *} \mathrm{P}<0.01$.

the effects of doxorubicin on apoptosis. As shown in Fig. 1B, these experiments confirmed that doxorubicin induces apoptosis in gastric cancer cells in a time- and dose-dependent manner. Moreover, these experiments showed that the differences in apoptosis among the 4 gastric cancer cell lines are not statistically significant $(\mathrm{P}>0.05)$.

Doxorubicin induces the phosphorylation of $p 38$ MAPK in human gastric cancer cells. The activation of the p38 MAPK pathway was shown to be involved in chemoresistance induced by doxorubicin (12). Therefore, the response of the MAPK signalling protein p38 on doxorubicin treatment was investigated by western blotting. The p38 MAPK protein level was affected by the treatment (Fig. 2A). Although the level of total p38 did not significantly change following a 24-h doxorubicin treatment, the level of phospho-p38 was markedly increased compared to untreated cells, in a time-dependent manner (Fig. 2A). Similar results were observed in three additional human gastric cancer cell lines (Fig. 2B). Overall, these results suggested that the high level of phospho-p38 may relate to the chemoresistance of gastric cancer cells to doxorubicin.

Inhibition of p38 MAPK reduces chemoresistance of gastric cancer cells to doxorubicin. In order to assess whether inhibition of p38 MAPK affects doxorubicin treatment in BGC823 and SGC7901 cells, the p38 MAPK inhibitor SB203580 was combined with doxorubicin to treat BGC823 and SGC7901 cells. The results from the MTT assay indicated that doxorubicin treatment significantly decreases survival of BGC823 and SGC7901 cells if it is combined with SB203580 (Fig. 3). Notably, this effect was dose-dependent. Overall, these results suggested that the inhibition of the p38 MAPK protein can sensitize gastric cancer cells to doxorubicin.

Inhibition of p38 MAPK enhances doxorubicin-induced apoptosis, increases the pro-apoptotic protein Bax level and significantly increases caspase-3 activity. To investigate the role of the p38 MAPK pathway in the development of doxorubicin resistance in gastric cancer cells, the SB203580 inhibitor was combined with doxorubicin to treat BGC823 cells. Flow cytometry analysis showed that cells treated with doxorubicin + SB203580 have a higher percentage of apoptosis compared to cells treated with doxorubicin alone $(\mathrm{P}<0.01)$ (Fig. 4A). The percentages of apoptotic cells were
$39.4 \pm 4.1 \%$ for the doxorubicin and $68.3 \pm 3.6 \%$ for the doxorubicin + SB203580 treatment. Fig. 4B shows the results of Hoechst staining, which confirmed the results of the flow cytometry analysis. Therefore, these experiments indicated that the p38 MAPK is involved in cell apoptosis induced by doxorubicin, and that inhibition of p38 MAPK enhances doxorubicin-induced apoptosis.

To further explore the mechanism by which SB203580 enhances doxorubicin-induced apoptosis in gastric cancer cells, we analyzed the expression of two proteins of the intrinsic mitochondrial apoptosis pathway, Bcl-2 and Bax. The pro-apoptotic Bcl-2 family member Bax is important for the initiation of mitochondrial dysfunction during apoptosis. Western blot analysis showed that the combination of SB203580 with doxorubicin increased the expression of Bax compared to untreated and doxorubicin-only treated cells. Moreover, the expression of the anti-apoptotic protein $\mathrm{Bcl}-2$ was reduced by treatment with the SB203580 + doxorubicin combination. We also detected the caspase-3 activity in cells treated with doxorubicin + SB203580. As shown in Fig. 4D, the caspase- 3 activity in these cells $(4.26 \pm 0.33)$ was increased by $\sim 239 \%$ compared to cells treated with doxorubicin $(1.78 \pm 0.28)$ or SB203580 (1.39 \pm 0.16$)$ alone. These results indicated that the mitochondria-mediated apoptotic pathway is involved in the inhibition of the p38 MAPK pathway induced by doxorubicin.

Inhibition ofp38MAPK increases chemosensitivity of BGC823 cells to doxorubicin in vivo. We showed that SB203580 can enhance doxorubicin-induced apoptosis in vitro, however, its in vivo effects remain unknown. Therefore, we used the human BGC823 cell line to establish a carcinoma xenograft model in athymic nude mice. Four experimental groups were examined: i) the control group (BGC823 cells treated with the vehicle solution, DMSO); ii) the doxorubicin group; iii) the SB203580 group and iv) doxorubicin + SB203580 group. As shown in Fig. 5, tumor volume data were plotted to compare the differences in the antitumor effect of different treatments in the course of the experiment (Fig. 5A). The combination of SB203580 and doxorubicin $\left(527 \pm 96 \mathrm{~mm}^{3}\right)$ markedly inhibited tumor growth, compared to no treatment in the control cells $\left(2,267 \pm 105 \mathrm{~mm}^{3}\right)$, and to doxorubicin $\left(1,256 \pm 107 \mathrm{~mm}^{3}\right)$ or SB203580 $\left(1,408 \pm 112 \mathrm{~mm}^{3}\right)$ treatment alone. There was no significant difference between animals treated with doxorubicin and SB203580. Furthermore, we found an increase 


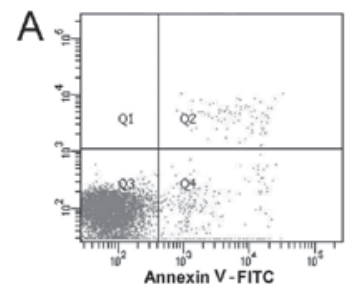

DMSO

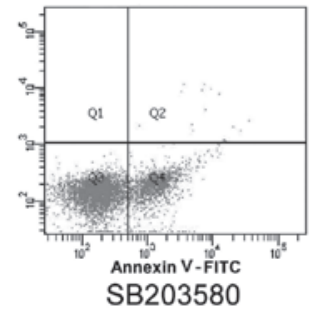

C

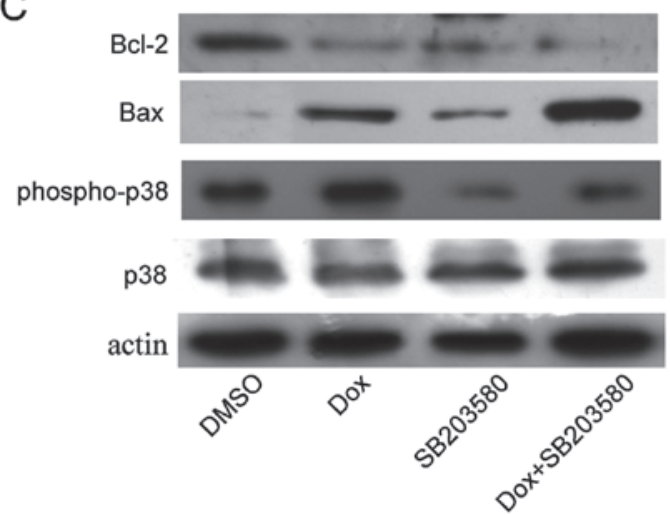

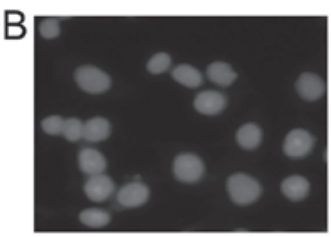

DMSO

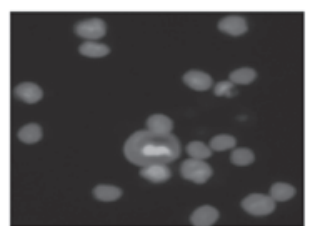

SB203580

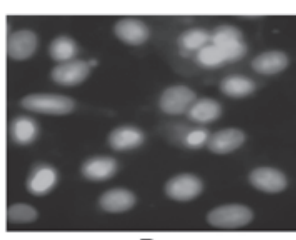

Dox

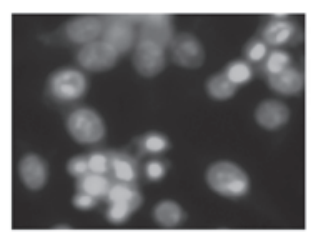

Dox+SB203580

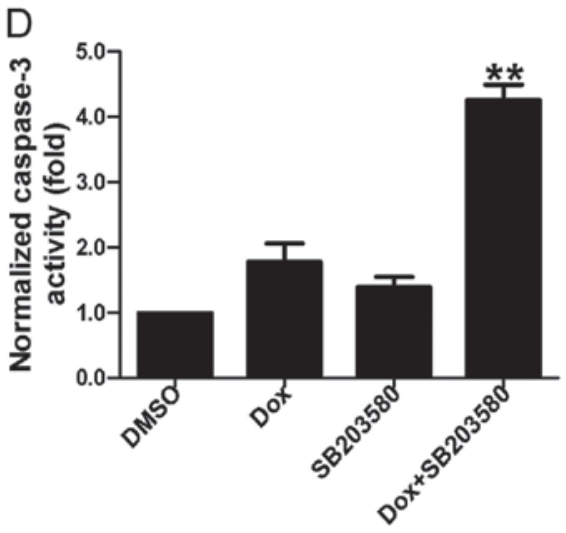

Figure 4. The combined effect of p38 mitogen-activated protein kinase (MAPK) inhibition and doxorubicin treatment ( $0.3 \mu \mathrm{M}, 12 \mathrm{~h})$ on gastric cancer cell apoptosis. (A) Flow cytometry analysis of apoptotic cells. DMSO (3.2 $\pm 1.1 \%$ ), cells incubated with $10 \mu \mathrm{M}$ dimethyl sulfoxide; Dox (39.4 $\pm 4.1 \%)$, cells treated with $0.3 \mu \mathrm{M}$ doxorubicin for $12 \mathrm{~h}$; SB203580 (15.4 $\pm 2.8 \%)$, cells pretreated with $20 \mu \mathrm{M} \mathrm{SB} 203580$ for $2 \mathrm{~h}$ followed by addition of $10 \mu \mathrm{M}$ DMSO; Dox + SB203580 $(68.3 \pm 3.6 \%)$, cells treated with $20 \mu \mathrm{M} \mathrm{SB} 203580$ followed by $0.3 \mu \mathrm{M}$ doxorubicin for $12 \mathrm{~h}$. FITC, fluorescein isothiocyanate. (B) Hoechst staining analysis of apoptotic cells (magnification, x400). DMSO, cells incubated with $10 \mu \mathrm{M}$ DMSO (2.4 $\pm 0.9 \%)$; Dox $(35.4 \pm 3.2 \%$ ), cells treated with $0.3 \mu \mathrm{M}$ doxorubicin for $12 \mathrm{~h}$;

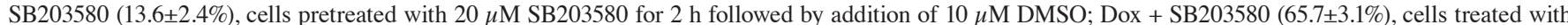
$20 \mu \mathrm{M}$ SB203580 followed by $0.3 \mu \mathrm{M}$ doxorubicin for $12 \mathrm{~h}$. (C) Western blot showing the levels of $\mathrm{p} 38 \mathrm{MAPK}$, pro-apoptotic and anti-apoptotic proteins. The p38, p-p38, Bax, Bcl-2 and actin bands were visualized by using polyclonal antibodies. Bcl-2, B-cell lymphoma 2; Bax, Bcl-2-associated X protein. (D) Normalized caspase-3 activity in the cells. DMSO, cells incubated with $10 \mu \mathrm{M}$ DMSO (1.0 $\pm 0.0 \%$ ); Dox (1.78 \pm 0.28$)$, cells treated with $0.3 \mu \mathrm{M}$ doxorubicin for $12 \mathrm{~h}$; SB203580 (1.39 \pm 0.16 ), gastric cancer cells pretreated with $20 \mu \mathrm{M} \mathrm{SB} 203580$ for $2 \mathrm{~h}$ followed by addition of $10 \mu \mathrm{M}$ DMSO; Dox + SB203580 (4.26 \pm 0.33 ), cells treated with $20 \mu \mathrm{M}$ SB203580 followed by $0.3 \mu \mathrm{M}$ doxorubicin for $12 \mathrm{~h}$. All experiments were performed in triplicate. ( ${ }^{* *} \mathrm{P}<0.01$ vs. Dox or SB203580).

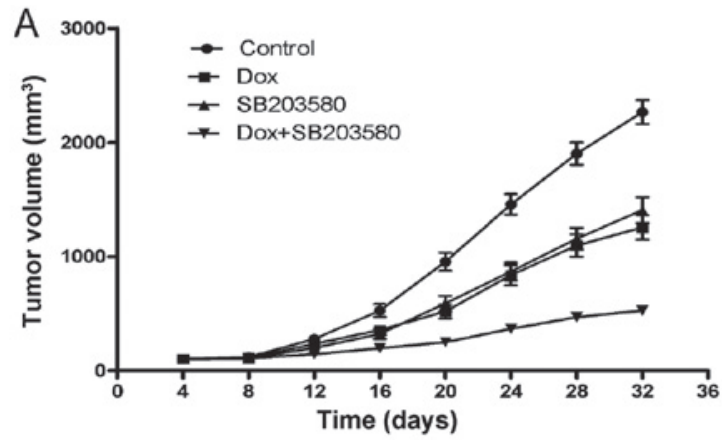

$\mathrm{B}$

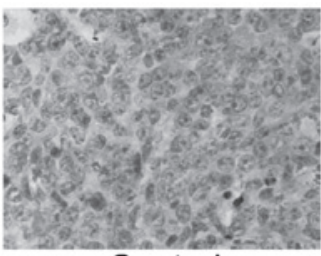
Control

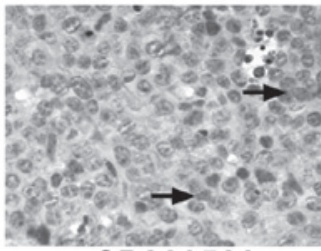

SB203580

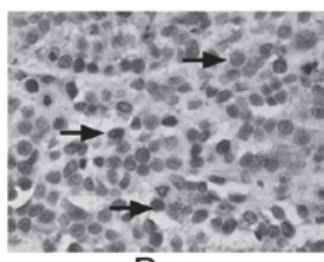

Dox

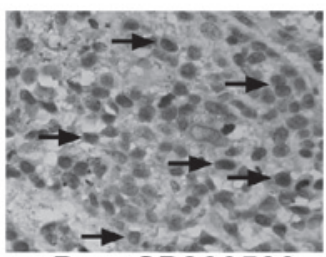

Dox+SB203580

Figure 5. The combination of SB203580 and doxorubicin (Dox) suppresses tumor growth in vivo. (A) Mean tumor volume \pm standard error of the mean (SEM) in nude mice of each group $(\mathrm{n}=6)$ at each time point. (B) Detection of apoptotic cells in tumor tissues with the terminal deoxynucleotidyl transferase-mediated dUTP nick end labeling (TUNEL) assay. Control, BGC823 cells treated with the vehicle solution; Dox, doxorubicin-treated cells (2 mg/kg), SB203580, SB203580-treated cells (10 mg/kg); Dox + SB203580, doxorubicin (2 mg/kg)- and SB203580 (10 mg/kg)-treated cells. Brown apoptotic cells are shown by the arrows. Magnification, $\mathrm{x} 400$. 
in the number of TUNEL-positive cells in the combined treatment group, as shown in Fig. 5B. The rate of apoptosis as estimated by the TUNEL assay in the three experimental groups was: $32.2 \pm 3.7 \%$ (doxorubicin), $12.8 \pm 2.5 \%$ (SB203580) and $58.6 \pm 3.9 \%$ (SB203580 + doxorubicin), respectively.

\section{Discussion}

In the past few decades, DNA-damaging agents, including doxorubicin, have been used in the clinic to treat cancer at the doses required to achieve efficacy $(13,14)$. However, the outcome of these treatments has proved unsatisfactory. One of the most important reasons for this failure is chemoresistance. A recent study showed that chemoresistance is the main obstacle in chemotherapeutic treatment of gastric cancer (15). A possible reason for chemoresistance is the failure of doxorubicin, when used alone, to cause cancer cell apoptosis, since the inactivation of apoptotic pathways following cellular stress is essential for cancer development (16). The mechanism by which a cell escapes apoptosis following stress, leading for example to DNA strand breaks, is assumed to involve intracellular signal transduction pathways (17). The MAPK pathway is considered an important intracellular signal transduction pathway in drug resistance (18).

It is known that the MAPK pathway is a major signal transduction cascade that ultimately triggers multiple cellular responses upon stimulation by chemotherapy (19). p38 MAPKs have been identified as a subfamily of MAPK. In the cells, the p38 MAPK pathway is primarily activated in response to environmental stress and is mainly involved in cell apoptosis and chemoresistance (11). It can be stimulated by various stresses, such as osmotic stress and heat shock, and by certain chemotherapeutic agents, for example doxorubicin (20). Recent studies confirmed that p38 MAPK activation is increased in gastric cancer $(12,21)$. Feng et al (12) reported increased resistance to chemotherapeutic agents in gastric cancer specimens expressing high levels of phospho-p38 compared with those expressing low levels of phospho-p38. Our results also confirmed that p38 MAPK activation relates to chemoresistance of gastric cancer cells to doxorubicin. However, no study to date has provided insights into the exact role of the p38 MAPK pathway in doxorubicin resistance of gastric cancer cells in vitro and in vivo.

In the present study, induced expression of phospho-p38 was observed following treatment with doxorubicin. Doxorubicin increased the level of phospho-p38 in a time-dependent manner. We next examined the expression of phospho-p38 in the presence and absence of the p38 MAPK inhibitor SB203580. SB203580 is a highly specific, potent, cell-permeable, selective, reversible, and ATP-competitive inhibitor of $\mathrm{p} 38$ MAPK (22). In doxorubicin-treated cells, relative inhibition of the phospho-p38 expression by SB203580 was estimated at $85 \%$. Our data showed that in BGC 823 cells, the induction of phospho-p38 expression was markedly blocked by SB203580, resulting in increased sensitivity of gastric cancer cells to the anticancer drug doxorubicin. When cancer cells were treated with doxorubicin + SB203580, the advantage of survival conferred by chemoresistance (p38 activation) disappeared and cell death was induced. In addition, we investigated the molecular events underlying cell death induced by doxorubicin and SB203580. An immunoblot assay was used to detect the level of phospho-p38, Bax and Bcl-2, and a colorimetric assay was used to measure the activity of caspase-3. These experiments confirmed that cell death may be associated with the increased expression of the pro-apoptotic protein Bax, and that doxorubicin treatment combined with inhibition of the p38 MAPK significantly increases the activity of caspase-3, which is the hallmark of cellular apoptosis.

$\mathrm{Bax}$ and $\mathrm{Bcl}-2$ are two main mitochondria-related Bcl-2 family protein members that mediate mitochondrial membrane permeability (23). Bax is a $23-\mathrm{kDa}$ protein and is considered a pro-apoptotic protein: it forms a transmembrane pore across the outer mitochondrial membrane, leading to loss of the membrane potential and enhanced apoptosis (24). $\mathrm{Bcl}-2$ is a $28-\mathrm{kDa}$ integral membrane oncoprotein that has anti-apoptotic properties, since it prevents pore formation and cell death (25). The ratio of Bcl-2 to Bax is a marker of cellular fate (26). When the ratio of Bcl-2 to Bax increases, caspase-3, the key executioner of apoptosis, is activated, and cells undergo apoptosis (27). This study showed that when cells were treated with doxorubicin combined with SB203580, the level of Bax and the caspase-3 activity were increased, and the level of Bcl-2 was decreased. The results suggested that treatment with doxorubicin and SB203580 induces Bax expression, which is expected to modulate the loss of mitochondrial membrane potential and contribute to the activity of caspase-3, and eventually, to gastric cancer cell death. Overall, inhibition of p38 MAPK enhanced doxorubicin-induced apoptosis, by activating the mitochondrial pathway of apoptosis.

In addition, our study showed that SB203580 can reduce p38 MAPK-mediated chemoresisitance in vivo, and does not enhance the toxicity of doxorubicin in nude mice. In the nude mice xenograft model, we found that the tumor size grew slowly and gradually increased at the final stages of the combined treatment with doxorubicin + SB203580. TUNEL staining showed that there are more apoptotic cells in mice treated with doxorubicin supplemented with SB203580, which suggests that decreased tumor growth rates are associated with an increase in apoptotic rate. All these results support our in vitro findings on cultured gastric cancer cell lines.

In conclusion, our data provide plausible evidence that SB203580 can act as a sensitizing agent for the conventional chemotherapeutic agent doxorubicin in the treatment of gastric cancer, both in vitro and in vivo, by attenuating the doxorubicin-induced chemoresistance. Standard chemotherapy combined with p38 inhibitors may thus represent a successful therapeutic strategy for the treatment of gastric cancer.

\section{Acknowledgements}

We thank Mr. Hong Xia at the Wuhan University for his outstanding administrative support in this study.

\section{References}

1. Kim J, Kim N, Park JH, Chang H, Kim JY, Lee DH, Kim JM, Kim JS and Jung HC: The effect of Helicobacter pylori on epidermal growth factor receptor-induced signal transduction and the preventive effect of celecoxib in gastric cancer cells. Gut Liver 7: 552-529, 2013. 
2. Jian P, Yanfang T, Zhuan Z, Jian W, Xueming Z and Jian N MMP28 (epilysin) as a novel promoter of invasion and metastasis in gastric cancer. BMC Cancer 11: 200, 2011.

3. Atmaca A, Pauligk C, Steinmetz K, Altmannsberger HM, Jäger E and Al-Batran SE: Prognostic impact of phosphorylated mitogenactivated protein kinase expression in patients with metastatic gastric cancer. Oncology 80: 130-134, 2011.

4. Oh SC: Update of adjuvant chemotherapy for resected gastric cancer. J Gastric Cancer 12: 3-6, 2012.

5. Kingma PS and Osheroff N: Topoisomerase II-mediated DNA cleavage and religation in the absence of base pairing. Abasic lesions as a tool to dissect enzyme mechanism. J Biol Chem 273: 17999-18002, 1998

6. Huang J, Ni J, Liu K, Yu Y, Xie M, Kang R, Vernon P, Cao L and Tang D: HMGB1 promotes drug resistance in osteosarcoma. Cancer Res 72: 230-238, 2012.

7. Wu K, Xie D, Zou Y, Zhang T, Pong RC, Xiao G, Fazli L, Gleave M, He D, Boothman DA and Hsieh JT: The mechanism of DAB2IP in chemoresistance of prostate cancer cells. Clin Cancer Res 19: 4740-4749, 2013

8. Yang YI, Lee KT, Park HJ, Kim TJ, Choi YS, Shih IeM and Choi JH: Tectorigenin sensitizes paclitaxel-resistant human ovarian cancer cells through downregulation of the Akt and $\mathrm{NF}_{\mathrm{K}} \mathrm{B}$ pathway. Carcinogenesis 33: 2488-2498, 2012.

9. Marengo B, De Ciucis CG, Ricciarelli R, Furfaro AL, Colla R, Canepa E, Traverso N, Marinari UM, Pronzato MA and Domenicotti C: p38MAPK inhibition: a new combined approach to reduce neuroblastoma resistance under etoposide treatment. Cell Death Dis 4: e589, 2013.

10. Cuenda A and Rousseau S: p38 MAP-kinases pathway regulation, function and role in human diseases. Biochim Biophys Acta 1773 $1358-1375,2007$

11. Keuling AM, Andrew SE and Tron VA: Inhibition of p38 MAPK enhances ABT-737-induced cell death in melanoma cell lines: novel regulation of PUMA. Pigment Cell Melanoma Res 23: 430-440, 2010

12. Feng R, Zhai WL, Yang HY, Jin H and Zhang QX: Induction of ER stress protects gastric cells against apoptosis induced by cisplatin and doxorubicin through activation of p38 MAPK. Biochem Biophys Res Commun 406: 299-304, 2011.

13. Harrington KJ, Rowlinson-Busza G, Syrigos KN, Vile RG, Uster PS, Peters AM and Stewart JS: Pegylated liposome-encapsulated doxorubicin and cisplatin enhance the effect of radiotherapy in a tumor xenograft model. Clin Cancer Res 6: 4939-4949, 2000.

14. Lluch A, Ojeda B, Colomer R, Barnadas A, Massuti B, Casado A, Angeles $\mathrm{C}$ and Maroto P: Doxorubicin and paclitaxel in advanced breast carcinoma: importance of prior adjuvant anthracycline therapy. Cancer 89: 2169-2175, 2000.

15. Yoshida M, Shimura T, Fukuda S, Mizoshita T, Tanida S, Kataoka H, Kamiya T, Nakazawa T,Higashiyama S and Joh T: Nuclear translocation of pro-amphiregulin induces chemoresistance in gastric cancer. Cancer Sci 103: 708-715, 2012.
16. Brown JM and Attardi LD: The role of apoptosis in cancer development and treatment response. Nat Rev Cancer 5: 231-237, 2005.

17. Cho SY, Jeong EM, Lee JH, Kim HJ, Lim J, Kim CW, Shin DM, Jeon JH, Choi K and Kim IG: Doxorubicin induces the persistent activation of intracellular transglutaminase 2 that protects from cell death. Mol Cells 33: 235-241, 2012.

18. Chung LY, Tang SJ, Sun GH, Chou TY, Yeh TS, Yu SL and Sun KH: Galectin-1 promotes lung cancer progression and chemoresistance by upregulating p38 MAPK, ERK, and cyclooxygenase-2. Clin Cancer Res 18: 4037-4347, 2012.

19. Li W and Melton DW: Cisplatin regulates the MAPK kinase pathway to induce increased expression of DNA repair gene $\mathrm{ERCCl}$ and increase melanoma chemoresistance. Oncogene 31: 2412-2422, 2012

20. Kubo T, Shimose S, Matsuo T, Sakai A and Ochi M: Efficacy of a nitrogen-containing bisphosphonate, minodronate, in conjunction with a p38 mitogen activated protein kinase inhibitor or doxorubicin against malignant bone tumor cells Cancer Chemother Pharmacol 62: 111-116, 2008.

21. Souma Y, Nishida T, Serada S, Iwahori K, Takahashi T, Fujimoto M, Ripley B, Nakajima K, Miyazaki Y, Mori M, et al: Antiproliferative effect of SOCS-1 through the suppression of STAT3 and p38 MAPK activation in gastric cancer cells. Int J Cancer 131: 1287-1296, 2012.

22. Li H, Wang X, Chen T and Qu J: p38 inhibitor SB203580 sensitizes the resveratrol-induced apoptosis in human lung adenocarcinoma (A549) cells. J Biochem Mol Toxicol 26: 251-257, 2012

23. Zamzami N, Brenner C, Marzo I, Susin SA and Kroemer G: Subcellular and submitochondrial mode of action of Bcl-2-like oncoproteins. Oncogene 16: 2265-2282, 1998.

24. Li X, Wu D, Shen J, Zhou M and Lu Y: Rapamycin induces autophagy in the melanoma cell line M14 via regulation of the expression levels of Bcl-2 and Bax. Oncol Lett 5: 167-172, 2013.

25. Scheel-Toellner D, Raza K, Assi L, Pilling D, Ross EJ, Lee WY, Curnow SJ, Buckley CD, Akbar AN, Lord JM and Salmon M: Differential regulation of nuclear and mitochondrial Bcl-2 in T cell apoptosis. Apoptosis 13: 109-117, 2008.

26. Kernt M, Arend N, Buerger A, Mann T, Haritoglou C, Ulbig MW Kampik A and Hirneiss C: Idebenone prevents human optic nerve head astrocytes from oxidative stress, apoptosis, and senescence by stabilizing BAX/Bcl-2 ratio. J Glaucoma 22 : 404-412, 2013

27. Shi L, Chen J, Yang J, Pan T, Zhang S and Wang Z: MiR-21 protected human glioblastoma U87MG cells from chemotherapeutic drug temozolomide induced apoptosis by decreasing Bax/Bcl-2 ratio and caspase-3 activity. Brain Res 1352: 255-264, 2010. 